American Journal of Nursing Science
2020; $9(3): 166-171$
http://www.sciencepublishinggroup.com/j/ajns
doi: 10.11648 /j.ajns.20200903.26
ISSN: 2328 -5745 (Print); ISSN: $2328-5753$ (Online)

\title{
Without Urinary Catheter During Perioperative Period of Microvascular Decompression of Trigeminal Neuralgia: The Inspiration Under the Concept of Rapid Recovery
}

\author{
Huixuan Chen ${ }^{1, \dagger}$, Cuiqing Liu ${ }^{3, \dagger}$, Wei Li ${ }^{1}$, Shiyong Wang', Weiju Chen ${ }^{2, *}$ \\ ${ }^{1}$ Department of Neurosurgery, The First Affiliated Hospital of Jinan University, Guangzhou, China \\ ${ }^{2}$ School of Nursing, Jinan University, Guangzhou, China \\ ${ }^{3}$ Department of Surgery, The First Affiliated Hospital of Jinan University, Guangzhou, China
}

Email address:

chenweiju@126.com (Weiju Chen)

${ }^{*}$ Corresponding author

$\dagger$ Huixuan Chen and Cuiqing Liu are co-first authors.

\section{To cite this article:}

Huixuan Chen, Cuiqing Liu, Wei Li, Shiyong Wang, Weiju Chen. Without Urinary Catheter During Perioperative Period of Microvascular Decompression of Trigeminal Neuralgia: The Inspiration Under the Concept of Rapid Recovery. American Journal of Nursing Science. Vol. 9, No. 3, 2020, pp. 166-171. doi: 10.11648/j.ajns.20200903.26

Received: April 29, 2020; Accepted: May 18, 2020; Published: May 27, 2020

\begin{abstract}
Objective: To determine the effect and advantages of no indwelling catheter during perioperative period of microvascular decompression for trigeminal neuralgia under the concept of rapid recovery. Methods: 30 patients underwent microvascular decompression for trigeminal neuralgia at our hospital during February 2017 and December 2019 were collected and divided into experimental group and control group by whether patients adopted urinary catheter or not at the concept of rapid recovery. The data of gender, age, operation time, intraoperative blood loss, urine tract infection, incidence of urinary retention, urinary comfort and average postoperative hospital stay were collected and compared. Results: No significant difference in gender, age, operation time, intraoperative blood loss $(P>0.05)$ between those two groups. The intraoperative fluid infusion and urine output in the control group were significantly higher than those in the experimental group. There was no significant difference in urine tract infection and urinary retention in these two groups $(P>0.05)$, comfort level $(P<0.05)$, and no significant difference in the average postoperative hospital stay $(P>0.05)$. Conclusions: Microvascular decompression for trigeminal neuralgia without urinary catheter during perioperative period under the concept of rapid recovery is feasible and safe, will not increase risk of urinary retention, improve postoperative comfort and shorten average postoperative hospital stay.
\end{abstract}

Keywords: Rapid Recovery, Trigeminal Neuralgia, Microvascular Decompression, Urinary Catheter, Nursing Stratege

\section{Introduction}

Trigeminal neuragia (TN) refers to an acute and electrical shock-like pain in area of the trigeminal nerve. Microvascular decompression (MVD) is a non-destructive surgical plan caused by $\mathrm{TN}$, and can relieve pain by separating the responsible vessels which pressed the trigeminal nerve root. It is effective and safe with low recurrence rate, and has been recognized as the preferred treatment $[1,2]$ for primary trigeminal neuralgia.

At present, there is no unified standard for the retention and removal time of urinary catheter in the concept of Enhanced recovery after surgery (ERAS), but early removal or no urinary catheter is the main trend of ERAS [3]. The traditional micro vascular for trigeminal neuralgia still use painless indwelling catheters, but studies have shown that with the development of enhanced recovery surgery, to shorten anesthesia and surgical time, control intraoperative blood loss, reduce intraoperative fluid volume and decrease urine output, there is no need to adot urinary catheters during the surgery. To further discuss the clinical advantages of no urinary catheter in patients undergoing microvascular decompression for trigeminal neuralgia, and to provide reference for the establishment of rapid recovery procedure, in this study, the clinical data of 30 patients with and without urinary catheter 
during perioperative period of micro vascular decompression for trigeminal neuralgia were analyzed. The results are as follows.

\section{Clinical Data}

From February 2017 to December 2019, 30 patients in the same neurosurgical group of our hospital underwent micro vascular decompression for trigeminal neuralgia were divided into experimental group (13 cases) and control group (17 cases) by whether adopting urinary catheter or not at the concept of rapid recovery out of the will of patients. Inclusion criteria: (i) meet the diagnose of primary trigeminal neuralgia (ii) volunteer to participate in this study. Exclusion criteria: (i) patients with diabetes, hypertension and coronary heart disease. (ii) patients with psychiatric history and cognitive impairment who can not cooperate. (iii) patients have dysuria caused by various kinds of serious urinary system diseases before surgery (including benign prostatic hyperplasia, urinary stone, infection and tumor, etc.). (iv) patients who had indwelled urinary catheter before surgery. Patients who meet the sampling criteria were grouped into study as per their wishes. Among the 30 patients, 13 were male and the rest 17 were female, aged 41-83 years old, hospitalized for 4-31 days. All patients complained of persistent or intermittent facial pain before operation for a period of 2-7 years in form of pricking or electrical shock-like pain. This study was approved by the Hospital Ethics Committee with the informed consent of the patients and their families.

\section{Methods}

Both groups underwent micro vascular decompression for trigeminal neuralgia, and the facial pain was stopped or alleviated to different extent after surgery.

\subsection{Group Status}

30 patients were divided into 13 in experimental group and 17 in control group. The control group adopted traditional perioperative management method: micro vascular decompression for trigeminal neuralgia under general anesthesia for endotracheal intubation, routine perioperative care, preoperative fasting for 8 hours, routine doctor advice and daily nursing work including admission education, pre-operation nursing, post-operation guidance and health education, etc. In the experimental group, the perioperative management was based on the concept of rapid recovery: conscious sedation and scalp nerve block anesthesia for trigeminal neuralgia micro vascular decompression, preoperative evaluation and education, treatment process and surgerial plan were explained to the patients, so that the patients knew their important role played in this plan. Preoperative fluid replacement method: oral carbohydrate drinks, usually $800 \mathrm{~mL}$ of $12.5 \%$ carbohydrate drank $10 \mathrm{~h}$ before surgery, and $\leq 400 \mathrm{~mL}$ [6] drank $2 \mathrm{~h}$ before surgery, to reduce preoperative thirst, hunger and irritability and the incidence of postoperative insulin resistance [7]. In this study,
$5 \%$ GNS500ML $+50 \%$ GS40ml was given orally $10 \mathrm{~h}$ before surgery, and $5 \% \mathrm{GS} 250 \mathrm{ml}$ was given $2 \mathrm{~h}$ before surgery.

\subsection{Optimal Management Plan of Urinary Catheter During Perioperative Period}

Bladder of both groups were emptied before entering the operation room. Control group: routine fluid replacement during surgery, catheter (14F Foley Catheter) inserted after anesthesia and extubated 1 day after surgery on the doctors' advice. Patients could urinate if they have urine after extubation with the family members' good cooperation. Experimental group: (i) emptied bladder before entering the operation room without catheter by diaper for urine. (ii) Objective-oriented fluid management during operation: avoid perioperative fluid overload and cardiopulmonary overload; after establishing hemodynamics monitoring (Stroke volume, cardiac output, systolic blood pressure variability, pulse pressure variability and stroke volume variability, etc.), rehydration tests based on 1-2 $\mathrm{ml} * \mathrm{~kg} / \mathrm{h}$ balanced salt crystalloid fluid were performed according to the monitoring index. Exampled with stroke volume, $200-250 \mathrm{ml}$ of colloidal fluid or balanced salt crystal fluid was given when stroke volume decreased; $200 \mathrm{ml}$ of fluid was added if the stroke volume increased by $10-15 \%$ or more; if the stroke volume increased less than $10 \%$, the rehydration test would stop and continue with basal infusion. Postoperation: No indwelling catheter, observe whether the bladder fills and the weight of diaper after returning to the ward. From the time the patient returned to the ward until the first urination, the nurses began to assess bladder fullness and whether the patient had a desire to urinate. Intermittent sterile catheterization was performed once for patients with urinary retention due to bladder fullness but unable to urinate spontaneously. Education to patients: (i) encourage to urinate spontaneously; (ii) try not to urinate in bed, urinate in the toilet with assistance; (iii) if there is a sense of urination but difficult to urinate, will induce urination with the family members' good cooperation.

\subsection{Observe Index}

\subsubsection{Time of Operation}

From the beginning to the end of anesthesia time. Intraoperative infusion volume: total infusion volume starts from anesthesia and ends upon operation. Intraoperative urine volume: All patients empty the bladder before entering the operating room. The total volume of urine for indwelling catheter group measured from indwelling catheter to end of operation, and judged by weight of diaper before and after operation for patients use diaper.

\subsubsection{Complication of Indwelling Catheter}

Urinary retention: Patients, averagely urinated within 3.5 hours in experimental group, who could not urinate if there is a sense of urination and could not urinate spontaneously after induce treatment; Patients in the control group, after extubating catheter, could not urinate if there is a sense of urination and could not urinate spontaneously after induce treatment, and should reindwell catheter [8] due to full 
bladder.

Urinary Tract Infection: Patients in the control group had midstream urine test after extubating catheter, and patients in the experimental group had midstream urine test after operation. According to the clinical diagnostic criteria of Diagnostic Criteria for Nosocomial Infection (Trial) in 2006, if the leukocyte in urine are $\geq 5 / \mathrm{HPF}$ in males or $\geq 10 / \mathrm{HPF}$ in females, with urinary tract irritation symptoms as frequent urination, urgent urination and painful urination, percussive pain in renal area or tenderness in lower abdomen, accompanying with or without fever, were diagnosed as urinary tract infection [9].

\subsubsection{Urination Comfort Score}

Visual simulation score (0-10) was applied to record the incidence and degree of pain according to patients' subjective feeling. 0 degree: No frequent urination, urgent urination, painful urination; I degree: Pain scores 1-3, accompanied with frequent urination, urgent urination, sense of urination; II degree: Pain scores 4-7, accompanied with frequent urination, urgent urination, sense of urination; III degree: Pain scores 8-10, accompanied with frequent urination, urgency, urinary sense [10].

\subsection{Statistic Analysis}

The data were displayed by average \pm standard deviation $($ mean $\pm \mathrm{SD})$, and Wilcoxon rank sum test was used for comparison between groups. The statistic test uses bilateral examination, and the standard level $\alpha=0.05$. The data were processed by SPSS20.0, $\mathrm{p}<0.05$ indicating the deviation has statistic value.

\section{Result}

\subsection{Clinical Information Comparison of Patients in Two Groups}

There was no significant difference in sex, age, operation time and intraoperative blood loss between the two groups (Table 1, $P>0.05$ ). The volume of intraoperative fluid infusion and urine output in the control group were significantly more than those in the experimental group. There was no significant difference in urinary tract infection, urinary retention $(P>0.05)$, comfort level $(P<0.05)$, no significant difference in postoperative average in-patient days $(P>0.05)$. See table 2 .

Table 1. Comparison of general data between the two groups.

\begin{tabular}{|c|c|c|c|}
\hline Clinical Information & Experimental Group $(n=13)$ & Control Group $(n=17)$ & $\boldsymbol{P}$ \\
\hline Sex (male/female) & $3 / 10$ & $13 / 4$ & 0.65 \\
\hline Age & $58.62 \pm 10.33$ & $68.71 \pm 9.78$ & 0.85 \\
\hline Intraoperative fluid infusion (ml) & $550.77 \pm 203.73$ & $1861.76 \pm 92.12$ & 0.00 \\
\hline Intraoperative blood loss (ml) & $88.46 \pm 21.15$ & $59.41 \pm 14.34$ & 0.08 \\
\hline Intraoperative urine output & 0 & $844.12 \pm 395.23$ & - \\
\hline
\end{tabular}

Table 2. Clinical observation between the two groups

\begin{tabular}{llll}
\hline Index Related & Experimental Group (n=13) & Control Group (n=17) \\
\hline Average catheter indwelling time (day)* & 0 & $3.29 \pm 1.04$ \\
Urinary tract infection & 0 & 0 & P \\
Urinary retention & 0 & 3 \\
Comfort level & & 1 \\
0 & 0 & 8 \\
I & 0 & 5 & 0.116 \\
II & 0 & 3 \\
III & 0 & $10.23 \pm 7.63$ \\
Average number of days in hospital after operation & $9.23 \pm 2.89$ & 0.05 \\
\hline
\end{tabular}

* No comparison between two groups

\subsection{Analysis on Urinary Retention and Urinary Tract Infection of Two Groups After Operation}

There was no significant difference in the incidence of urinary retention between the experimental group $(0 \%)$ and the control group $(17 \%)(P=0.116,>0.05)$. In the experimental group, 13 cases had no urinary retention and all of them urinated by themselves, while in the control group, 2 cases out of the 17 cases had urinary retention after extubation of catheter and urinated by themselves after one intermittent catheterization. The incidence of urinary tract infection was 0 , but there was no significant difference between the two groups $(P=0.050$, Table 2$)$.

\subsection{The Postoperative Comfort level and Average Length of Hospital Stay of the Two Groups}

The postoperative comfort level 0 degree was significantly higher in the experimental group (13 cases) than in the control group (1 case). The urethral symptoms (Grade I, Grade II, Grade III) in the control group were significantly higher than those of patients without indwelling catheter, there were significant difference in comfort level between two groups ( $p$ $<0.05$ ). The average length of hospital stay after operation was shortened by 5 days, but no significant difference between the two groups $(\mathrm{p}>0.05)$. 


\section{Discussion}

Fast-track surgery (FTS), also known as Enhanced Recovery After Surgery (ERAS), refers to the combination of optimization measures for perioperative treatment with evidence-based medical evidence, and the adoption of an optimized clinical path through multidisciplinary teamwork, in order to reduce the physical and psychological trauma stress of surgical patients, reduce complications and shorten hospital stays, and accelerate the recovery of patients [11]; FTS was first advocated by Danish physician Kehlet $[12,13]$, and has been widely used in countries around the world [14-18].

A goal-oriented restrictive volume therapy strategy can maintain a balance between access and intake, avoid too much or too little infusions, reduce the load on the heart, lungs and kidneys, and reduce the patient's urine output during the operation, which may be one of the reason for no urination in the experimental group. In recent years, the domestic ERAS concept has been applied to Thoracic Surgery [19], Gastrointestinal Surgery [20], Urology [21, 22], Obstetrics and Gynecology [23], Orthopedics [24-26] and other fields with good clinical effect. Accelerated Rehabilitation Surgery Chinese Expert Consensus and Path Management Guide (2018 Edition) advocates the concept and measures of goal-directed fluid therapy (GDFT) to guide fluid therapy. The goal of ERAS fluid management is try to minimize changes in body fluid volume. Insufficient capacity can lead to insufficient perfusion and organ dysfunction, and water and sodium retention is the main reason for postoperative intestinal paralysis and related complications. Therefore, the balance fluid should be used to maintain the balance of amount in and out during the operation, to avoid excessive and insufficient infusion [11]. In this study, we adopted a goal-oriented restrictive volume treatment strategy to maintain the balance of input and output and to avoid to much or too little infusion, emptied the bladder before surgery, used diapers during surgery, and the operation time in the experimental group was 2. 5-4 hours. None of the observed cases urinated during operation. A goal-oriented restrictive volume therapy strategy to reduce the load on the heart, lungs, and kidneys may be one of the reasons to avoid patients urinate and indwelling catheters during surgery, and further research is needed for confirmation.

At present, no indwelling urinary catheter during perioperative period has been used in other neurosurgery operations [27], which shows that no indwelling catheter can not only improve patients' postoperative comfort, but also shorten the average hospital stay, which is consistent with the results of this study. In related research [28], trigeminal neuralgia has applied the rapid recovery concept combined with the clinical path during the perioperative period, but still uses painless indwelling catheter during operation, which is removed 2-3 hours after the operation. This will increase the cost of patients and lower patients' comfort. This study suggests that trigeminal neuralgia microvascular decompression surgery is safe and feasible without urinary catheters throughout the perioperative period, and will further improve patients' comfort, shorten hospital stay, reduce hospitalization costs, and increase patients' satisfaction.

Urinary catheter management is one of the management core of rapid rehabilitation. Indwelling urinary catheters during surgery can lead to intraoperative blood pressure and heart rate increase [29], urinary tract infection, urinary retention [30], urethral irritation, dysuria [31] and other related complications. Indwelling catheters $>2$ days can significantly increase the incidence of urinary tract infections. Alyami etc.[32] reported 65 patients undergoing elective colon surgery had unconventional indwelling catheters after surgery with a urinary retention rate of $9.0 \%$ and a urinary tract infection rate of $1.5 \%$. In the sample included in this study, the experimental group had no urinary catheter throughout the perioperative period bur used diapers to receive urine during the operation; and the urinary catheter was indwelled after anesthesia in the control group and was extubated after 2-5 days of operation following the doctors' advice. The rate of urinary retention in the control group was $11 \%$, which was almost consistent with the results of Alyami and other studies. The incidence of urinary tract infection in the control group was inconsistent with the results of Alyami and other studies, which may be related to a fewer of sample size.

The difference in the average length of hospital stay between the two groups of patients in this study was relatively meaningless, but the average length of hospital stay in the experimental group was 5 days less than that in the control group. The main reason is that the experimental group adopted the concept of rapid rehabilitation to avoid complications after general anesthesia. Two patients developed pulmonary infections in the control group, which led to longer hospital stays.

\section{Conclusion}

Under the concept of rapid rehabilitation, patients of trigeminal neuralgia microvascular decompression applied no indwelling catheter during perioperative period have not increase the risk of urinary retention, and can reduce the incidence of urinary retention and urinary tract infection, improve postoperative comfort, shorten postoperative average hospital stay, reduce hospital costs and improve patients' satisfaction. However, the current study is only a single-center study, multi-center, multi-angle and large sample studies are still needed to confirm.

\section{Acknowledgements}

The authors declare no conflict of interest. Thanks to the supervision of the study from the continuing nursing research group of "Nursing Research and Knowledge Transformation" alliance of Guangdong-Hong Kong-Macao Greater Bay Area. 


\section{References}

[1] Chai Y, Chen M, Zhang W, et al. Predicting the outcome of microvascular decompression for primary trigeminal neuralgia by the use of magnetic resonance tomographic angiography [J]. J Craniofac Surg, 2013, 24 (5): 1699-1702.

[2] Revuelta-Gutierrez R, Martinez-Anda J J, Coll J B, et al. Efficacy and safety of root compression of trigeminal nerve for trigeminal neuralgia without evidence of vascular compression [J]. World Neurosurg, 2013, 80 (3-4): 385-389.

[3] Wu Q, Sha Y S. Application Status and Progress of Rapid Rehabilitation Concept in Surgical Nursing [J]. Journal of Nurse Training, 2018, 33 (20): 1850-1853.

[4] Yang M, Chen J, Che G W, et al. Cost-benefit analysis of perioperative urethral indwelling in patients with lung cancer lobectomy $[\mathrm{J}]$. Chinese Clinical Journal of Thoracic and Cardiovascular Surgery, 2016, 23 (05): 421-424.

[5] Cao Z Q, Shi J, Jin H T, et al. Urethra affects the rehabilitation of pediatric appendicitis after laparoscopic surgery [J]. Domestic and Overseas Medical Research, 2019, 17 (05): 18-19.

[6] Feldheiser A, Aziz O, Baldini G, et al. Enhanced Recovery After Surgery (ERAS) for gastrointestinal surgery, part 2: consensus statement for anaesthesia practice $[\mathrm{J}]$. Acta Anaesthesiologica Scandinavica, 2016, 60 (3): 289-334.

[7] Zheng X L, Luo Z Z, Cai J Y, et al, Effect of rapid rehabilitation surgery concept on perioperative insulin resistance and inflammatory response in patients with partial hepatectomy [J]. Guangdong Medical, 2017, 38 (05): 709-712.

[8] Stallard S, Prescott S. Postoperative urinary retention in general surgical patients [J]. Br J Surg, 1988, 75 (11): 1141-1143.

[9] Nicolle L E. Urinary catheter-associated infections [J]. Infect Dis Clin North Am, 2012, 26 (1): 13-27.

[10] Wang G D, Wang M F. Comparison on comfort of urinary catheter in gynecological surgery patients before and after anesthesia [J]. Chinese Journal of Nursing, 2005 (09): 705-706.

[11] Chen L, Chen Y J, Dong H L, et al. Chinese consensus and clinical guidelines for Enhanced Recovery After Surgery (2018 editon) [J]. Chinese Journal of Practical Surgery, 2018, 38 (01): $1-20$.

[12] Kehlet H, Wilmore D W. Multimodal strategies to improve surgical outcome [J]. Am J Surg, 2002, 183 (6): 630-641.

[13] Wilmore D W, Kehlet H. Management of patients in fast track surgery [J]. BMJ, 2001, 322 (7284): 473-476.

[14] Kosar S, Seelen H A, Hemmen B, et al. Cost-effectiveness of an integrated 'fast track' rehabilitation service for multi-trauma patients involving dedicated early rehabilitation intervention programs: design of a prospective, multi-centre, non-randomised clinical trial [J]. J Trauma Manag Outcomes, 2009, 3: 1 .

[15] Mazza F, Venturino M, Turello D, et al. Enhanced recovery after surgery: adherence and outcomes in elderly patients undergoing VATS lobectomy [J]. Gen Thorac Cardiovasc Surg, 2020 .
[16] Alimena S, Falzone M, Feltmate C M, et al. Perioperative glycemic measures among non-fasting gynecologic oncology patients receiving carbohydrate loading in an enhanced recovery after surgery (ERAS) protocol [J]. Int J Gynecol Cancer, 2020.

[17] Melloul E, Lassen K, Roulin D, et al. Guidelines for Perioperative Care for Pancreatoduodenectomy: Enhanced Recovery After Surgery (ERAS) Recommendations 2019 [J]. World J Surg, 2020.

[18] Ghodoussipour S, Naser-Tavakolian A, Cameron B, et al. Internal audit of an enhanced recovery after surgery protocol for radical cystectomy [J]. World J Urol, 2020.

[19] Che G. [Current Situation and Strategy of Day Surgery in Patients with Lung Cancer by Enhanced Recovery after Surgery] [J]. Zhongguo Fei Ai Za Zhi, 2020, 23 (1): 1-4.

[20] Ni C Y, Wang Z H, Huang Z P, et al. Early enforced mobilization after liver resection: A prospective randomized controlled trial [J]. Int J Surg, 2018, 54 (Pt A): 254-258.

[21] Hu G W, Wang H M. Application of Accelerated Rehabilitation Surgery in Perioperative Nursing of Sun's End-Flexible Ureter Nephroscope Holmium Laser Lithotripsy [J]. Electronic Journal of Clinical Medical Literature, 2018, 5 (44): 129-131.

[22] Liu Q. Effect of fast recovery surgical nursing on postoperative rehabilitation of patients undergoing urological surgery $[\mathrm{J}]$. Nursing Practice and Research, 2017, 14 (15): 58-59.

[23] Zhang J J, Zhang Y, Ma Q. Analysis of the influence of eating time on gastrointestinal peristalsis function, adverse reactions and blood sugar after gynecological malignant tumors after laparotomy [J]. Journal of Nurses Training, 2019, 34 (16): $1512-1515$.

[24] Zhao C M, Jin C M, Shao Y. Clinical study on the time of first meal after orthopedic spinal surgery of patients undergoing general anesthesia [J]. Chongqing Medical, 2019, 48 (18): 3224-3227.

[25] Yin M, Yan Y, Fan Z, et al. The efficacy of Enhanced Recovery after Surgery (ERAS) for elderly patients with intertrochanteric fractures who received surgery: study protocol for a randomized, blinded, controlled trial $[\mathrm{J}]$. J Orthop Surg Res, 2020, 15 (1): 91.

[26] Jiang H H, Jian X F, Shangguan Y F, et al. Effects of Enhanced Recovery After Surgery in Total Knee Arthroplasty for Patients Older Than 65 Years [J]. Orthop Surg, 2019, 11 (2): 229-235.

[27] Wu M H, Chen M J, Liu W J. Study on the effect of no indwelling of urinary catheter in patients with Chiari malformation during perioperative under accelerated rehabilitation surgery mode $[\mathrm{J}]$. Journal of Sichuan University (Medical Science Edition), 2018, 49 (4): 670-672.

[28] Zhao J, Xie X H. Application of clinical nursing path combined with rapid rehabilitation surgery concept in perioperative period of patients with trigeminal neuralgia $[\mathrm{J}]$. Tofay Nurse (later issue), 2017 (12): 49-51.

[29] Mai H Z. Analysis of the effect of Dacronine hydrochloride on the discomfort of male patients with indwelling urinary catheter under general anesthesia [J]. China Practical Medical, 2018 (35): 125-126. 
[30] Lei Y, Liu J Q, Song S S. Effect study of nursing project on reducing the incidence of urinary retention after extubation in gynecological patients with indwelling catheter [J]. Electronic Journal of Clinical Medical Literature, 2018 (51): 139-140.

[31] Li B, Wang F. Discussion on standard construction of urinary catheter removal in patients with lower limb fracture after spinal anesthesia under accelerated rehabilitation surgery mode [J]. Chinese Medicine and Clinic, 2018 (05): 860-862.

[32] Alyami M, Lundberg P, Passot G, et al. Laparoscopic Colonic Resection Without Urinary Drainage: Is It "Feasible"?[J]. J Gastrointest Surg, 2016, 20 (7): 1388-1392. 